\title{
CÓMO ESTRUCTURAN EL CONTENIDO DE LA ESCRITURA ALUMNOS DE EDUCACIÓN PRIMARIA
}

\author{
José Luis Gallego Ortega \\ Universidad de Granada
}

\begin{abstract}
RESUMEN: En este artículo se presenta una investigación cuyo principal objetivo fue conocer el proceso de estructuración del contenido de la escritura por alumnos de educación primaria. En la investigación, de carácter exploratorio y cualitativo, se recurre al "estudio de caso colectivo" y se utiliza la "entrevista cognitiva" para la obtención de datos, aplicando el "análisis de contenido" en la interpretación de los mismos. En ella se han identificado las operaciones que tienen lugar durante la estructuración del contenido, la forma de ejecutar dichas operaciones por estos alumnos y las principales dificultades encontradas. Los resultados de la investigación revelan que, aunque estos alumnos suelen realizar las operaciones que conlleva el proceso de estructuración textual, muestran dificultades relevantes en cuanto a la estructuración general de las ideas en un texto. Finalmente, se ofrecen algunas orientaciones generales para la enseñanza de la estructuración del contenido de la escritura en este nivel educativo.
\end{abstract}

PALABRAS CLAVE: Procesos cognitivos de la escritura, estructuración de la escritura, operaciones de la estructuración, educación primaria.

\section{HOW PRIMARY EDUCATION STUDENTS ORGANIZE THE PLANNING OF THE WRITTEN}

\begin{abstract}
The present paper deals with a study aimed at providing an insight into how students with Primary Education plan their written expression. The exploratory and qualitative investigation resorts to the "collective case study" and it is used the "cognitive interview" to obtain the items, applying the "content analysis" in the interpretation of them. This research has allowed us to identify the process in content organization, how students make these operations and the main difficulties founded in it. The results of the research show that, although these students normally make the operations of this textual organization process, they have important difficulties regarding the general
\end{abstract}


planning of the ideas in a text. Finally, this article gives some guidelines for the teaching of writing structure in Primary Education students.

KEYWORDS: Writing cognitive processes, writing structure, operations of the organization, primary education.

Recibido: $24 / 05 / 2011$

Aceptado: 22/09/2011

\section{INTRODUCCIÓN}

En este artículo se presentan resultados parciales, derivados de un macro-proyecto de investigación (Necesidades educativas especiales en la expresión escrita de alumnos, en contextos de atención a la diversidad), financiado por el Ministerio de Ciencia y Tecnología, durante el trienio 2001-2004 (BSO2000-1203). El objetivo central de la investigación era analizar la expresión escrita de los alumnos en tres dimensiones: 1) los procesos cognitivos y metacognitivos, implicados en la expresión escrita; 2) las características del texto elaborado, tanto en el nivel macro-estructural (cohesión textual) como en el micro-estructural (ortografía, formación de signos -letras, palabras- etc.); 3) los contextos de escritura. No obstante, este estudio sólo incluye los resultados obtenidos del análisis de una de las operaciones cognitivas más relevantes de la planificación de la composición escrita, como es la estructuración textual.

La expresión escrita representa un reto importante para profesores y alumnos, y su aprendizaje es fundamental para el éxito académico de los estudiantes. La escritura, tradicionalmente relegada en la investigación educativa, adquiere un relevante protagonismo en los últimos años, como pone de manifiesto el Handbook of Research on Teaching (Richardson, 2001), en el que definitivamente es considerada como un importante campo de estudio, y, de forma especial, el Handbook of Writing Research (McCarthur, Graham y Fitzgerald, 2006), donde se aprecia claramente la evidente atención que la escritura recibe por parte de los investigadores.

Sin embargo, las investigaciones sobre la escritura en el contexto español han sido escasas, aunque en las últimas décadas se hayan realizado meritorios estudios. En efecto, la literatura sobre la expresión escrita revela la existencia de diversos estudios los cuales se han centrado en diferentes aspectos o dimensiones del proceso escritor, tomando como referente diferentes grupos de sujetos. Así, algunas investigaciones se han ocupado tanto de la evaluación de las habilidades escritoras de los alumnos, identificando sus principales dificultades (Camps y Ribas, 2000; Ramos, Cuadrado e Iglesias, 2005), como del diseño de programas para paliar dichas dificultades (Lecuona, 1999; García y Fidalgo, 2008). Otros estudios han señalado cuáles son las disfunciones escritoras de los alumnos que presentan dificultades de aprendizaje (Salvador, 2000; García y Bausela, 2004). Finalmente, existen algunos trabajos, similares al que se presenta, en los que se plantean objetivos relacionados con los procesos psicológicos implicados en la composición escrita, cuyo referente han sido también diversos grupos de sujetos (Vilà, 1998; Arroyo y Salvador, 2005; Lecuona, Rodríguez y Sánchez, 2003; Gutiérrez y Salvador, 2006; Salvador y García, 2007; 
Gallego, 2008a, 2008b; Gallego y Rodríguez, 2009) y en los que se subraya la complejidad que encierra este aprendizaje y la conveniencia de su estudio.

No obstante, si a esta complejidad intrínseca se le une la dificultad de indagar en el conocimiento de los procesos cognitivos que intervienen en la escritura, se justifica que esta investigación acote su campo de estudio y analice solo uno de los procesos cognitivos que supuestamente los alumnos activan a la hora de componer sus textos: la estructuración del contenido, como proceso inherente a la fase de planificación del texto, que es, sin duda, uno de los procesos centrales de la escritura, junto con la autorregulación (Hayes y Nash, 1996). Además, la relevancia de este proceso cognitivo obedece a diferentes razones (Fitzgerald y Teasly, 1986): a) la organización textual es vista como una faceta de la calidad y coherencia de una composición escrita; b) la adecuada organización es un signo de la coherencia de la historia en su conjunto; c) el conocimiento de la estructura textual involucra conocimientos sobre cómo son conectadas temporal y causalmente las partes de la historia.

\section{OBJETIVOS E INTERROGANTES BÁSICOS}

En esta investigación, de carácter eminentemente cualitativo, se pretende pues evaluar el proceso cognitivo de la estructuración de contenido en la composición escrita de alumnos de $6^{\circ}$ curso de Educación Primaria (EP), para identificar posibles dificultades y sugerir propuestas de mejora. El estudio está orientado hacia los siguientes objetivos: 1) Determinar qué habilidades poseen alumnos de $6^{\circ}$ curso de Primaria para estructurar el contenido de sus producciones escritas; 2) Evaluar el proceso cognitivo de la estructuración del contenido de la escritura por estos alumnos e identificar posibles disfunciones; 3) Proponer orientaciones didácticas para la mejora.

Debido al carácter exploratorio de la investigación, y de acuerdo con los objetivos planteados, las hipótesis iniciales se formulan en forma de interrogantes: 1) ¿Los alumnos de $6^{\circ}$ curso de EP conocen y utilizan adecuadamente el proceso de estructuración textual?; 2) ¿Qué estrategias cognitivas activan estos alumnos en la estructuración del contenido de un texto?; 3) ¿Cuáles son las principales dificultades que encuentran en dicho proceso?

\section{CONTEXTO TEÓRICO Y DE INVESTIGACIÓN}

En la actualidad, la escritura se concibe como un proceso de comunicación, de reflexión y de resolución de problemas, en el que es preciso obtener y preparar las ideas para luego desarrollarlas en un contexto y con una intención comunicativa determinada. Esto implica reflexionar acerca de lo que se quiere escribir, sobre los pensamientos y conocimientos del tema, sobre la situación de comunicación y sobre las ideas a expresar, a la vez que involucra la realización de esquemas, redacción de borradores, volver al texto, releer, corregir y reformular lo que se escribe reiteradamente (Smith, 1982).

La escritura es, pues, una actividad compleja que depende de la conquista de múltiples habilidades y de la propia capacidad de quien escribe para coordinar diversos procesos. En efecto, el aprendizaje de la expresión escrita demanda inicialmente en el alumno la conquista de ciertas habilidades mecánicas (o de bajo nivel cognitivo) de la 
escritura (caligrafía, ortografía, recuperación léxica) hasta desarrollar otras habilidades de mayor nivel cognitivo (planificación, revisión, conocimiento metacognitivo). De ahí que diferentes investigaciones hayan puesto de manifiesto que la enseñanza tradicional de la expresión escrita no propicia el desarrollo de estrategias efectivas para su aprendizaje (Troia, 2002), aun cuando la escritura es la principal herramienta para que los alumnos demuestren sus conocimientos (Graham y Harris, 2005). Sin embargo, el auge cada vez mayor del enfoque comunicativo de la lengua ha contribuido al desarrollo de nuevos planteamientos didácticos, cuyo propósito es capacitar al alumno para adquirir una eficaz competencia comunicativa.

En perspectiva histórica, se pueden identificar diferentes modelos teóricos para la enseñanza de la composición escrita. Entre ellos, destaca el modelo cognitivo de Flower y Hayes (1981), posteriormente modificado y actualizado por Hayes (1996), en el que se inscribe esta investigación, por ser considerado en la actualidad como el modelo más potente y que mejor explica la escritura de textos, al concebirla como un proceso de resolución de problemas en el que el escritor activa estrategias de planificación, análisis e inferencia (Cfr. Figura 1). El modelo representa una descripción organizada de la estructura de la composición escrita, superando la concepción lineal y unidireccional de la producción escrita y poniendo de manifiesto las interrelaciones que en ella se gestan: la interrelación del contexto social (audiencia, colaboradores) con el contexto físico (texto elaborado) (Álvarez y Ramírez, 2006).

Flower y Hayes elaboraron un modelo centrado en la identificación y descripción de los procesos que configuran el proceso global de la composición escrita, en el que contemplan tanto los procesos específicos para la construcción del texto (planificación, análisis, revisión, etc.) como otros procesos mentales que intervienen en la composición escrita (memoria a corto plazo, memoria a largo plazo, creatividad...) y admiten que las disfunciones detectadas en las composiciones escritas de los alumnos derivan, en buena medida, de las dificultades para ejecutar dichos procesos (Troia, 2002).

No obstante, el foco de atención de este modelo es el sujeto, su memoria de trabajo, su capacidad para hacer eficientes los procesos de codificación y decodificación de la información, así como otros aspectos o componentes referidos a la persona que escribe: la afectividad, la motivación, los procesos cognitivos -planificación, transcripción y revisión- y la memoria a largo plazo.

La planificación o reflexión (Hayes, 1996) implica la representación mental, más o menos completa, de lo que el escritor quiere hacer y de cómo lo va a realizar. En esta fase se establecen los objetivos del texto y el plan global que orientará toda la producción. Incluye, a su vez, tres subprocesos u operaciones: 1) la génesis de contenidos (ideas), que supone la búsqueda de informaciones, ya sean perfectamente estructuradas o no, en la memoria a largo plazo. 2) la organización y estructuración de contenidos, o sea, una vez que las ideas se han obtenido, corresponde a otro subproceso la estructuración y organización de las mismas en una estructura global, con el fin de otorgar una mayor coherencia al texto escrito. 3) la formulación de objetivos, es decir, es necesario determinar los objetivos del texto, para controlar el acto de la composición. 


\section{CONTEXTO DE LA TAREA}

CONTEXTO SOCIAL

\begin{tabular}{c}
\hline Audiencia \\
Colaboradores \\
\hline
\end{tabular}

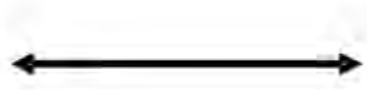

SUJETO
CONTEXTO FISICO

Textos escritos

Medios de escritua

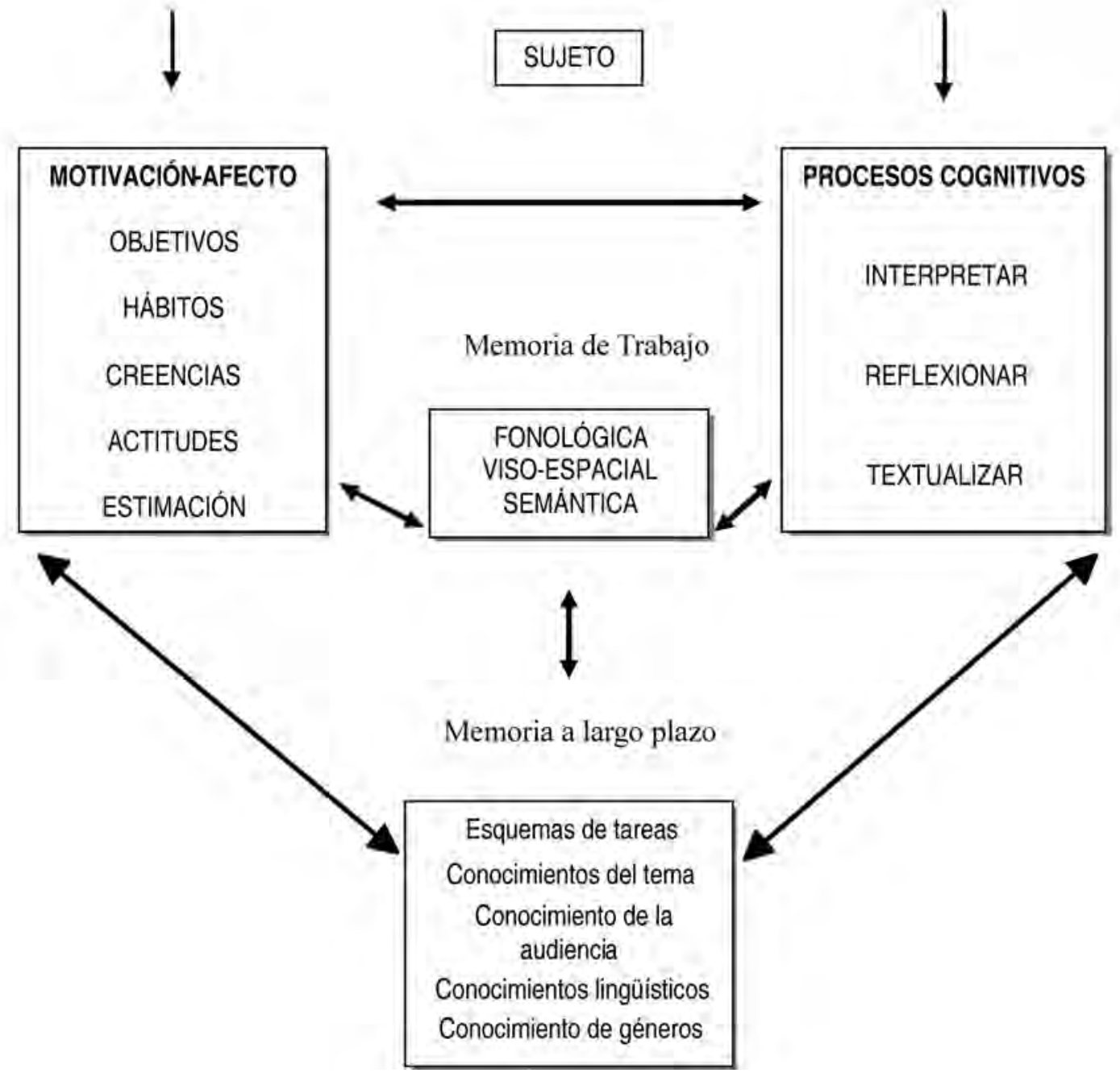

Figura 1. Modelo de Hayes (1996) 
La transcripción o producción del texto (Hayes, 1996) supone la transformación de las ideas del escritor en lenguaje visible e inteligible para el lector. Es un proceso muy complejo, ya que el escritor debe atender a varias demandas conjuntamente: Ios propósitos y el contenido del texto, la construcción morfosintáctica, la ejecución gráfica de las letras, etc. La selección del contenido apropiado y del estilo depende de la idea que se tenga de los posibles lectores (Hedge, 1998). El propósito comunicativo o resultado que el escritor espera lograr al componer su texto sería imposible determinarlo sin un conocimiento previo de quién leerá el texto. A este tipo de conocimiento, Tribble (1996) lo denomina conocimiento del contexto. Además, para Flower y Hayes (1981), el propósito de la comunicación y los procesos que se desarrollan con ese fin están condicionados por dos tipos de objetivos: procedimentales, que instruyen acerca de cómo realizar el proceso de escritura y de contenido, que especifican todo lo que el escritor quiere decir a sus lectores.

La revisión implica un análisis consciente de lo planificado y escrito, e incluye, a su vez, dos subprocesos: 1) la evaluación del resultado y 2) la revisión y corrección de lo producido. La función de evaluación es fundamental para identificar y analizar los problemas del texto (Hayes, 1996). Resulta ampliamente reconocido que los escritores competentes no concluyen sus escritos en un primer intento sino a través de sucesivas versiones, hasta la producción de la versión definitiva.

De otra parte, en la investigación sobre la composición escrita, se han identificado tres enfoques principales, cuya diferencia reside, principalmente, en el objeto de estudio (Castelló y Monereo, 1996; Salvador, 2008): producto, proceso y ecológico. El enfoque de producto, de orientación conductista, constituye la perspectiva más tradicional en el estudio de la escritura y analiza principalmente los aspectos formales (grafía y ortografía) y estructurales del texto (significado del texto y cohesión textual).

El enfoque de proceso, centrado en las habilidades (cognitivas, emocionales y motivacionales) que se activan durante la construcción de un texto, está orientado al conocimiento, desarrollo y funcionamiento de los procesos cognitivos implicados en la escritura. Este enfoque, de orientación cognitiva, forma parte de los planteamientos que subyacen en la investigación mediacional-cognitiva o interpretativa. La trascendencia de este enfoque, por la relevancia de sus resultados, resulta muy atractiva para la investigación, dado que permite el estudio de tales procesos. Este es el enfoque de investigación sobre la escritura predominante.

En el enfoque ecológico, la atención se centra en el contexto en el que se produce el aprendizaje y su finalidad es indagar acerca de la función social y cultural del texto para reinterpretar el lenguaje escrito. Su objetivo último es evaluar la efectividad de los programas de intervención.

Ahora bien, en esta investigación, se adopta el enfoque de proceso -asumiendo el cambio de perspectiva que se ha producido en el estudio de la escritura en las últimas décadas-, por su innegable relevancia para comprender en profundidad los procesos ejecutivos de la escritura, dado su carácter explicativo e interpretativo, y pese a la complejidad intrínseca que supone para los investigadores. 


\section{MÉTOdo}

\section{Sujetos}

Se ha trabajado con una muestra de 12 alumnos (5 niños y 7 niñas) de $6^{\circ}$ curso de EP, cuyas edades oscilan entre los 11 y los 12 años (media de 11.07), que están escolarizados en un centro público urbano. Todos ellos obtienen buenos resultados académicos y poseen un buen nivel de adaptación personal y social. Ninguno presenta dificultades en el aprendizaje ni alteraciones físicas o psíquicas.

Se ha adoptado la estrategia metodológica del "estudio de caso colectivo", ya que aporta gran cantidad y calidad de información y se considera pertinente para asegurar la comprensión de este tipo de problemas (Stake, 1998). Los sujetos son considerados, pues, como un único caso aunque ello no excluye la posibilidad de detectar algunas diferencias intracaso. Por ello, lo esencial de esta investigación no es la amplitud de la muestra, sino el análisis en profundidad del caso investigado (McMillan y Schumacker, 2005).

\section{Obtención y análisis de datos}

Para la obtención de datos, se ha optado por la entrevista semiestructurada de tipo cognitivo, mediante la cual el investigador interroga al sujeto sobre su percepción acerca de los procesos cognitivos implicados en la estructuración textual, inmediatamente después de haber elaborado un texto narrativo. La entrevista, que se realizó a partir de un cuestionario (Cfr. Salvador, 2005, 62-66), elaborado y validado previamente según el procedimiento de "juicio de expertos" y "triangulación" (Fox, 1981; Hodder, 2000), se desarrolló en un lugar apropiado del centro escolar, exento de ruidos y de elementos distractores, en horario escolar, y evitando ocupar el tiempo de recreo o actividades en las que los niños estuviesen especialmente motivados. Se procuró asimismo un ambiente distendido y agradable en todas las entrevistas.

El cuestionario-guía, del que se reproduce un extracto del apartado referido a la estructuración de contenido en el texto (Cfr. Cuadro 1), pretende ayudar al alumno a reflexionar y verbalizar lo que hace inmediatamente después de enfrentarse a la elaboración de un texto narrativo, para inducir los procesos desarrollados en la composición escrita y las posibles relaciones entre ellos (concretamente, en este caso, sobre el proceso de estructuración de contenido).

La narración de una historia exige habilidades de procesamiento ejecutivo: organización, planificación, memoria de trabajo o procesos de análisis-síntesis (Barkley, 1997), y su elección se justifica por múltiples razones: a) la narración es uno de los modos del discurso monogal con más arraigo en la mente humana y que funciona como esquema de comprensión y de interpretación más básico (Calsamiglia, 2000); b) la producción narrativa involucra múltiples funciones cognitivas (Gonçalves et al., 2002), así como diferentes aspectos afectivos, emotivos, éticos y estéticos (González Landa, 2009); c) es útil para comprender el procesamiento cognitivo de los niños (Miranda, García y Soriano, 2005); d) es el texto más frecuente en los escritos que elaboran los alumnos libremente (Salvador, 2008) y un recurso relevante para el dominio de la competencia comunicativa de los niños (De Amo y Ruiz, 2006). 
Cuadro 1. Resumen del Cuestionario-guía sobre estructuración de contenido

- ¿Antes de escribir un texto o una redacción piensas qué tipo de texto vas a escribir, por ejemplo, narrativo, descriptivo, argumentativo, diálogo?

- ¿Ordenas las ideas y las palabras de forma distinta según el tipo de texto que vas a escribir?

a) Cuando describes un objeto o una escena ¿sigues el orden: arriba-abajoderecha-izquierda?

b) Cuando describes un suceso ¿lo ordenas en el tiempo según la secuencia principio, medio y fin?

c) ¿Señalas el problema, las razones y la solución, cuando escribes un texto para convencer a otro de algo?

d) ¿Señalas lo que es igual y es diferente cuando comparas dos objetos o sucesos?

e) Cuando escribes una noticia o un reportaje ¿distingues lo más importante de lo menos?

- ¿Sigues alguna regla que te ayude a recordar las partes del texto?

- ¿Utilizas algún esquema o cuadro para organizar las ideas que se te van ocurriendo para escribir un texto o una redacción?

- Antes de escribir un texto o una redacción ¿clasificas u ordenas de alguna forma las ideas (palabras) que vas a escribir?

- ¿Por qué las ordenas así y no de otra forma?

- ¿Piensas las frases completas o las escribes como se te van ocurriendo en ese momento?

Por ello, para la realización de esta tarea, se pidió a cada alumno que relatara por escrito un cuento o historia sobre algún acontecimiento, hecho o suceso de su interés, sin ninguna restricción temporal, con la intención de que, inmediatamente después de haber elaborado el texto, fuese capaz de evocar durante la entrevista las operaciones realizadas durante su construcción. Todas las entrevistas fueron grabadas y transcritas literalmente. De esta forma, el discurso oral se transformó en texto escrito sobre el que se realizó el análisis de contenido. Los "protocolos" en los que se recogen las reflexiones de los alumnos se han sometido al análisis de contenido (Bardín, 1986; Krippendorff, 2002), estableciendo un sistema de categorías para inferir cuáles son los procesos cognitivos activados, durante la elaboración de un texto, de acuerdo con el modelo teórico antes descrito.

De esta forma, las dimensiones psicolingüísticas implicadas en la estructuración textual se agruparon, para facilitar el análisis y la interpretación de los datos, en aquellas categorías que se corresponden con las operaciones que los alumnos realizan durante la estructuración del contenido de un texto. En efecto, la consideración de la estructuración de contenido, a partir del modelo teórico descrito (Flower y Hayes, 1981; Hayes, 1996) -ampliamente reconocido por la comunidad científi- 
ca-, como un proceso en el que el alumno crea y organiza, de forma coherente y cohesionada, la estructura de una composición (es decir, la capacidad del alumno para estructurar de forma adecuada la organización formal y de contenido de un texto escrito) permite establecer dos categorías u operaciones básicas (Cfr. Tabla 1), en cuya definición se incorporan sus matices.

Tabla 1. Operaciones de la estructuración del contenido de la escritura

\begin{tabular}{|l|l|}
\hline \multicolumn{2}{|l|}{ LA ESTRUCTURACIÓN COMO PROCESO COGNITIVO } \\
\hline Categorías [códigos (+-E)] & Definición \\
\hline Estructuración general (E1) & $\begin{array}{l}\text { Capacidad del sujeto para organizar previamente las ideas en } \\
\text { un texto }\end{array}$ \\
\hline Estructuración textual (E2) & $\begin{array}{l}\text { Capacidad del sujeto para adecuar las ideas al tipo de texto } \\
\text { que quiere construir }\end{array}$ \\
\hline
\end{tabular}

Este sistema de categorías también fue debidamente validado por el procedimiento de "juicio de expertos" y "triangulación", de tal manera que, según los expertos, reúne las cualidades o requisitos necesarios para satisfacer las exigencias planteadas en esta investigación (Bardín, 1986):

- Exhaustividad: cualquier unidad puede ser ubicada en una categoría, es decir, las categorías establecidas permiten clasificar toda la información recogida en las entrevistas.

- Exclusión mutua: cada unidad de registro se incluye en una sola categoría, de tal manera que no pueda pertenecer a más de una.

- Homogeneidad: el principio de exclusión mutua depende de la homogeneidad de las categorías, esto es, las categorías están definidas de acuerdo con un mismo principio de definición.

- Objetividad: las categorías resultan inteligibles para diferentes codificadores.

- Pertinencia: las categorías son relevantes y adecuadas a los objetivos del estudio.

- Productividad: las categorías proporcionan resultados ricos en índices de inferencias, en hipótesis nuevas, en datos fiables.

\section{Resultados}

Los resultados sobre el proceso de estructuración se obtienen del análisis de contenido de las entrevistas, así como de la cuantificación (frecuencias positivas/negativas, que se corresponden con la ejecución/no realización o inadecuación de la operación demandada) y del análisis cualitativo de las respuestas dadas a las cuestiones planteadas. Sin embargo, lo relevante de la investigación no es la cuantificación de los datos sino el análisis cualitativo, en profundidad, de las entrevistas. 
No obstante, en el análisis cuantitativo (Cfr. Tabla 2) se aprecian valores significativamente más elevados de frecuencias positivas que de negativas en las dos categorías, de lo cual puede inferirse:

Tabla 2. Frecuencias de las categorías de estructuración de contenido

\begin{tabular}{|l|c|c|c|c|c|}
\hline $\begin{array}{l}\text { CATEGORÍAS DE } \\
\text { ESTRUCTURACIÓN }\end{array}$ & Códigos & $\begin{array}{c}\text { Frecuencias } \\
\text { positivas }\end{array}$ & $\begin{array}{c}\text { Frecuencias } \\
\text { negativas }\end{array}$ & $\begin{array}{c}\text { Frecuencias } \\
\text { totales }\end{array}$ & $\begin{array}{c}\text { Porcentajes } \\
\text { de frecuencias }\end{array}$ \\
\hline Estructuración general & +E1/-E1 & 46 & 17 & 63 & 37.95 \\
\hline Estructuración textual & +E2/-E2 & 87 & 16 & 103 & 62.05 \\
\hline \multicolumn{2}{|l|}{ TOTAL DE FRECUENCIAS } & 133 & 33 & 166 & 100 \\
\hline
\end{tabular}

1. Que los alumnos entrevistados realizan las operaciones que conlleva el proceso de estructuración del contenido de la composición escrita en el $80.1 \%$ de los casos.

2. Que los alumnos entrevistados no realizan, o lo hacen de forma inadecuada, en un $19.9 \%$ de los casos, alguna de las operaciones implícitas en el proceso de estructuración del contenido de la composición escrita.

3. Que la operación cognitiva en la que los alumnos entrevistados encuentran mayores problemas es en la estructuración general de las ideas del texto.

4. Que, en general, las operaciones implícitas en la estructuración del contenido de la escritura son asequibles para estos alumnos.

Se ha de insistir, sin embargo, para la correcta interpretación de los datos, que, al tratarse de un estudio de caso colectivo, el número de frecuencias establecido se refiere al conjunto de los doce sujetos que conforman la muestra y no a un sujeto solo. Pero, como ya se ha señalado, el interés de este estudio no reside en la interpretación cuantitativa de los datos, sino en el análisis cualitativo de las entrevistas. Para ilustrar esta interpretación, se incluyen algunas expresiones literales de los alumnos entrevistados, referidas a las categorías contempladas en el proceso de estructuración del contenido de la composición escrita.

\section{Sobre la estructuración general (+E1/-E1)}

El análisis cualitativo de las entrevistas pone de manifiesto que la mayoría de los sujetos piensa las frases completas antes de escribirlas en sus textos y después transcribe lo que ha pensado, aunque, a veces, las respuestas no son tan nítidas.

P: ¿Piensas las frases completas o las escribes como se te van ocurriendo?

R: Es preferible ordenarlas en la mente, agrupar las palabras para que luego se entienda mejor, para que quede mejor el texto (ES9, ES10, ES11). 
R: Primero voy pensando la frase completa y después voy y la escribo (ES4, ES5, ES8, ES12).

R: Completas. Algunas veces todo seguido (ES2, ES3).

$\mathrm{R}$ : Todo seguido (ES1).

En efecto, los alumnos plasman, en ocasiones, las ideas en el texto de forma organizada, a pesar de que sus respuestas admitan, a veces, que ignoran el uso de esta estrategia.

P: Antes de escribir un texto o una redacción ¿clasificas u ordenas de alguna forma las palabras que vas a escribir?

R: Sí, primero hago el esquema, la introducción (ES9, ES12).

R: Sí, pienso muy bien lo que voy a poner (ES5, ES8; ES10, ES11).

$\mathrm{R}$ : Yo escribo lo que se me va ocurriendo (ES1, ES2).

$\mathrm{R}:$ No, pues no (ES3).

Rara vez se ha podido apreciar que los alumnos recurran a algún esquema, cuadro o truco que les facilite la elaboración de un texto.

P: ¿Utilizas algún truco para organizar las ideas que se te van ocurriendo?

$\mathrm{R}$ : A veces hago un esquema para guiarme (ES11, ES12)

$\mathrm{R}$ : Como se me van ocurriendo en ese momento (ES1, (ES3).

$\mathrm{R}: \mathrm{No}(\mathrm{ES} 2, \mathrm{ES} 4, \mathrm{ES} 6)$.

$\mathrm{R}:$ No, no es necesario (ES1, ES3).

\section{Sobre la estructuración textual (+E2/-E2)}

El análisis cualitativo de las entrevistas revela que los alumnos, en general, conocen la estructura de diferentes textos (narrativo, argumentativo...) y defienden que, en función del tipo de texto que van a realizar, ordenan las palabras y las ideas de forma distinta. En este sentido, los alumnos admiten que: a) tienen en cuenta la secuencia temporal de principio, medio y final; b) explican el problema, las razones y la solución al mismo; c) diferencian los hechos principales de los secundarios; d) siguen un orden en las descripciones de objetos; e) señalan lo que es igual y diferente, cuando comparan dos objetos o sucesos entre sí.

P: ¿Antes de escribir un texto o una redacción piensas qué tipo de texto vas a escribir?

R: Sí, primero lo pienso (ES4, ES5, ES6, ES8, ES9, ES10, ES11, ES12).

Algunos, incluso, matizan su respuesta indicando la razón por la cual tienen en cuenta el tipo de texto que escriben.

P: ¿Antes de escribir un texto o una redacción piensas qué tipo de texto vas a escribir?

R: Sí, porque son textos distintos (ES6, ES10, ES11, ES12).

R: Sí, porque si no no se entendería (ES5, ES9).

R: Hay que cuidar el orden (ES4, ES8). 
Otros alumnos, por el contrario, indican que no tienen en cuenta el tipo de texto que van a escribir.

P: ¿Antes de escribir un texto o una redacción piensas qué tipo de texto vas a escribir?

$\mathrm{R}:$ No (ES1, ES3).

R: Yo lo escribo poco a poco y me va saliendo (ES1).

Sin embargo, algunos alumnos, aun admitiendo que conocen la existencia de diferentes tipos de texto, señalan que, en el momento de escribir, tienen dudas acerca de la ordenación de las ideas según el tipo de texto, no piensan en el texto concreto que van a elaborar, ni recurren a reglas mnemónicas que les faciliten esa tarea.

P: ¿Ordenas las ideas y las palabras de forma distinta según el tipo de texto que vas a escribir?

R: Sí (ES9, ES11, ES12); a veces sí (ES1); creo que sí (ES3, ES4, ES5, ES6, ES8).

$\mathrm{P}:$ ¿Sigues alguna regla que te ayude a recordar las partes del texto?

R: No, sobre la marcha (ES3).

R: Pongo lo más importante al principio, no me hace falta (ES1).

\section{DisCUSIÓN Y CONCLUSIONES}

Se debe insistir en el carácter exploratorio de este estudio y en que los resultados han de ser interpretados a la luz de varias limitaciones (tamaño de la muestra, selección no aleatoria de los participantes y peculiaridades intrínsecas a todo estudio de caso). Pese a lo cual, de los resultados obtenidos en esta investigación, es posible hacer modestas aportaciones al conocimiento de uno de los procesos cognitivos de la escritura más relevantes (la estructuración) por alumnos de $6^{0}$ curso de EP. En efecto, este estudio de caso permite señalar inicialmente dos conclusiones generales: a) los alumnos de la muestra ejecutan, aunque no de forma sistemática, las operaciones cognitivas que conlleva la estructuración del contenido en la escritura, cuando se enfrentan a la elaboración de un texto; b) existen, no obstante, algunas dificultades, referidas al proceso de estructuración textual, que han de ser atendidas convenientemente en el aula para mejorar el proceso general de la composición escrita.

Sin embargo, teniendo en cuenta los objetivos de este estudio, se ha de dar respuesta a las tres cuestiones planteadas inicialmente, para determinar las principales aportaciones de esta investigación.

1. ¿Los alumnos de $6^{\circ}$ curso de EP conocen y utilizan el proceso de estructuración de contenido?

En general, el estudio revela que los alumnos poseen suficiente conocimiento del proceso de estructuración de contenido, aunque no utilicen siempre de forma adecuada las estrategias que les permiten organizar, de forma coherente y cohesionada, un texto escrito. Este hallazgo, al contrario de lo que otras investigaciones han sugerido (Lecuona et al., 2003; Gallego y Rodríguez, 2009), es coincidente con los datos obtenidos por Vilà (1998), en un estudio realizado con una muestra de alumnos 
escolarizados en el ciclo superior de la E.G.B. y también con los descubrimientos realizados en otras investigaciones previas, realizadas con muestras de sujetos de características similares a las de este estudio (Arroyo y Salvador, 2005; Gallego, 2008b). Curiosamente los resultados de nuestro estudio también son coincidentes con los datos obtenidos en otra investigación previa, en la que se trabajó con alumnos de educación secundaria con discapacidad auditiva (Gutiérrez y Salvador, 2006). Sin embargo, los datos obtenidos son discrepantes con los hallazgos de otras investigaciones, en las que se trabajó con muestras de sujetos afectados por parálisis cerebral (Salvador y García, 2007) o retraso mental Gallego, 2008a).

\section{2. ¿Qué estrategias cognitivas activan durante la estructuración de un texto?}

El estudio pone de manifiesto, asimismo, que estos alumnos recurren al uso de diferentes estrategias cognitivas básicas cuando se enfrentan a la estructuración del contenido de un texto. En efecto: a) generalmente, los alumnos clasifican y ordenan las ideas que van a plasmar en un texto; b) frecuentemente, los alumnos piensan las frases $u$ oraciones que con posterioridad incluirán en sus textos; c) en general, los alumnos estructuran el contenido de sus escritos en función del tipo de texto que van a escribir; d) comúnmente se aprecia un mayor despliegue de habilidades, en cuanto a la organización del contenido, según la estructura textual. Estos datos corroboran, aunque con algunos matices, los hallazgos obtenidos en otras investigaciones, realizadas con grupos de sujetos diferentes (Vilà, 1998; Arroyo y Salvador, 2005; Salvador y García, 2007, Gallego, 2008b; Gallego y Rodríguez, 2009).

Sin embargo, los datos de este estudio no son consistentes con los resultados de investigaciones anteriores, en las que se muestran limitaciones en cuanto a la secuenciación y organización de las ideas en el texto, que los autores atribuyeron a su déficit lingüístico y experiencial (Gutiérrez y Salvador, 2006).

Se ha de subrayar, asimismo, que no se han apreciado diferencias significativas en cuanto al género. Ello es coherente con estudios que evidencian la inexistencia de diferencias significativas entre chicos y chicas en la composición de una redacción (Ramos, Cuadrado e Iglesias, 2005) y también corrobora en cierta medida los datos obtenidos en un reciente estudio (Jones y Myhill, 2007) en el que se explican las escasas diferencias de género halladas en las características lingüísticas de la escritura de alumnos de educación secundaria; aunque no sea nueva la impresión de que las alumnas son más eficaces que los alumnos en las actividades lingüísticas, ni tampoco que las razones aducidas para justificar esa idea sean muy dispares (véanse, entre otros, Browne, 1994; Millard, 1997; Demie, 2001; Barrs y Pidgeon, 2002; Ramos, Cuadrado e Iglesias, 2005; Peterson y Kennedy, 2006; Berninger et al., 2008).

\section{3. ¿Qué dificultades encuentran estos alumnos en dicho proceso?}

Finalmente, este estudio revela las principales dificultades de los alumnos durante la estructuración del contenido de un texto, que básicamente coinciden con los resultados obtenidos en otros estudios, realizados con diferentes muestras (Vilà, 1998; Lecuona et al., 2003; Arroyo y Salvador, 2005; Gutiérrez y Salvador, 2006; Salvador y García, 2007; Gallego, 2008a, 2008b; Gallego y Rodríguez, 2009). Es 
decir: a) descuidan la búsqueda de información y la organización de las ideas de un texto; b) desatienden las tipologías textuales a la hora de elaborar un escrito, aunque admiten tenerlas en cuenta; c) escasa iniciativa para desplegar habilidades en la organización general de las ideas en el texto. Asimismo, otros estudios (Graham, 1990; Graham y Harris, 1996 y 1997; Wong et al., 1997; Salvador, 2000; García y Bausela, 2004) encontraron problemas en alumnos con dificultades de aprendizaje a la hora de estructurar y organizar la información escrita, y algunos autores lo atribuyeron a un mal uso de las estrategias de recuperación de información (Graham, 1990). Además, en este estudio, la impresión que otorga a las alumnas mayores cotas de eficacia que a los alumnos en las actividades lingüísticas se pone en entredicho, aunque el discurso de género, como sugieren algunos autores (Cameron, 2005; Jones y Myhill, 2007), es poco útil como lente para considerar la escritura de chicos y chicas.

\section{Proyección didÁctica}

Es evidente que la calidad de un texto depende, entre otras cosas, de la adecuada organización de sus ideas y del respeto a su propia estructura, lo que conlleva necesariamente un conocimiento previo de las estructuras textuales y el despliegue de numerosas habilidades lingüísticas. A este efecto, la bibliografía especializada ha puesto de manifiesto que un método efectivo para ayudar a los estudiantes a mejorar sus composiciones escritas es instruirles en el planeamiento de estrategias de planificación, redacción, revisión y autorregulación, además de ser importante que el instructor dé instrucciones claras y supervise directamente el trabajo de los aprendices para proporcionarles feedback acerca de la exactitud en el uso de la estrategia, durante la realización de las tareas.

Abordar la composición escrita como una habilidad de carácter complejo, procesal, interactivo, recursivo en su funcionamiento interno y auto-regulado, aconseja un entrenamiento estratégico con el fin de contribuir a un aprendizaje más eficiente, a lo que también coadyuva el conocimiento de los procesos y subprocesos de la comunicación escrita. En este sentido, la enseñanza de estrategias se percibe como una ayuda para los estudiantes, por cuanto les permite enriquecer y aumentar sus habilidades como escritores, proporcionándoles nuevas y diferentes maneras de estructurar y formular su prosa (Tracy, Reid y Graham, 2009) e incrementándoles la competencia escritora (De la Paz y Graham, 1997).

Por consiguiente, pese a que la enseñanza de la escritura es una tarea tan compleja como la escritura misma, a partir de la bibliografía especializada sobre la enseñanza de estrategias efectivas en la planificación (estructuración) de la escritura, y en sintonía con los resultados y conclusiones de este estudio, se sugieren las siguientes tareas para facilitar el aprendizaje de esta habilidad: 1) Potenciar la motivación hacia el lenguaje escrito, proporcionando a los alumnos elogios o reafirmaciones tangibles de forma frecuente e intermitente (talleres de escritura, ambientes estimulantes...); 2) Confeccionar fichas o tarjetas, en las que se ilustren los pasos para planificar y organizar un tema de escritura; 3) Elaborar borradores previos sobre temas de interés, que, después, se utilizarán en la redacción de los textos; 4) Utilizar programas informáticos, en los que se pueden plasmar las ideas iniciales de un texto, 
que posteriormente pueden ser utilizadas durante la elaboración de éste; 5) Mostrar ejemplos de escritura y dialogar sobre ellos.

No obstante, el carácter inicial de este estudio abre nuevos interrogantes de especial interés para la investigación en este campo. Los resultados están abiertos a futuras comprobaciones o réplica, pudiéndose completar el estudio con muestras más numerosas y/o analizando otros procesos cognitivos o utilizando diferentes tipos de texto.

\section{REFERENCIAS BIBLIOGRÁFICAS}

ÁLVAREZ, T. y RAMÍREZ, R. (2006). Teorías o modelos de producción de textos en la enseñanza y el aprendizaje de la escritura. Didáctica. Lengua y Literatura, 18, 29-60.

ARROYO, R. y SALVADOR, F. (2005). El proceso de planificación en la composición escrita de alumnos de Educación Primaria. Revista de Educación (Madrid), 336, 353-376.

BARDíN, L. (1986). Análisis de contenido. Madrid: Akal.

BARKLEY, R. A. (1997). ADHD and the nature of self-control. New York: Guildford Press.

BARRS, M. y PIDGEON, S. (2002). Boys and writing. London: Centre for Literacy in Primary Education [CLPE].

BERNINGER, V. W.; NIELSEN, K. H.; ABBOTT, R. D.; WIJSMAN, E. y RASKIND, W. (2008). Gender differences in severity of writing and reading disabilities. Journal of School Psychology, 46(2), 151-172.

BROWNE, A. (1994). The content of writing in the early years: issues of gender. Reading, 28(3), 2-8.

CALSAMIGLIA, H. (2000). Estructura y funciones de la narración. Textos, 25, 9-21.

CAMERON, J. (2005). Language, gender and sexuality: current issues and new directions. Applied Linguistics, 26(4), 482-502.

CAMPS, A. y RIBAS, T. (2000). La evaluación del aprendizaje de la composición escrita en situación escolar. Madrid: CIDE.

CASTELLÓ, M. y MONEREO, C. (1996). Un estudio empírico sobre la enseñanza y el aprendizaje de estrategias para la composición de textos argumentativos. Infancia y Aprendizaje, 74, 39-55.

DEMIE, F. (2001). Ethnic and gender differences in educational achievement and implications for school improvement strategies. Educational Research, 43, 91-106.

DE AMO, J. M. y RUIZ, Ma M. (2006). Los géneros discursivos en la educación lingüística: la narración y la descripción. Lenguaje y Textos, 23-24, 9-29.

DE LA PAZ, B. y GRAHAM, S. (1997). Strategy instruction in planning: effects on the writing performance and behavior of students with learning difficulties. Excepcional Children, 63, 167-181.

FITZGERALD, J. y TEASLY, A. B. (1986). Effects of instruction in narrative structure on children's writing. Journal of Educational Psychology, 78(6), 424-432.

FLOWER, L. y HAYES, J. R. (1981). A cognitive process theory of writing. College Composition and Communication, 32(4), 365-387. 
FOX, D. J. (1981). El proceso de investigación en educación. Pamplona: Eunsa.

GALLEGO, J. L. (2008a). La planificación de la expresión escrita por alumnos con retraso mental. Revista de Educación (Madrid), 346, 267-290.

GALLEGO, J. L. (2008b). La planificación de la expresión escrita por alumnos de educación primaria. Bordón, 60(2), 63-76.

GALLEGO, J. L. y RODRÍGUEZ, A. (2009). La estructuración del contenido de la escritura por alumnos con discapacidad visual. Revista de Ciencias de la Educación (Madrid), 220, 445-466.

GARCÍA, J. N. y BAUSELA, E. (2004). Relación del conocimiento de la persona, de la tarea y de la estrategia en relación a la escritura con la coherencia y productividad de la composición escrita en niños-as con y sin dificultades de aprendizaje y-o bajo rendimiento. Revista de Educación (Madrid), 334, 223-234.

GARCÍA, J. N. y FIDALGO, R. (2008). Instrucción de la auto-regulación y el autoconocimiento en la composición escrita. Barcelona: Davinci.

GONZÁLEZ LANDA, Ma C. (2009). Aproximación al leer y al escribir como procesos interactivos y situados. Didáctica. Lengua y Literatura, 21, 157-190.

GONÇALVES, O. F.; MACHADO, P. P.; KORMAN, Y. y ANGUS, L. (2002). Narrative analysis and psychopathology. En L. Beutler y M. Marlik (ed.), Rethinking the DSM. Washington, DC: APA Press.

GRAHAM, S. (1990). The role of production factors in learning disabled student's compositions. Journal of Educational Psychology, 82, 781-791.

GRAHAM, S. y HARRIS, K. (1996). Self-regulation and strategy instruction for students who find writing and learning challenging. En C. M. Levy y S. R. Ransdell (eds.), The Science of Writing. Hillsdale, NJ: Lawrence Erlbaum Associates, pp. 347-360.

GRAHAM, S. y HARRIS, K. (1997). It can be taught, but it does not develop naturally: myths and realities in writing instruction. School Psychology Review, 26(3), 14-24.

GRAHAM, S. y HARRIS, K. (2005). Improving the writing Performance of Young Struggling Writers: Theoretical and Programmatic Research From the Center on Accelerating Student Learning. The Journal of Special Education, 39(1), 19-33.

GUTIÉRREZ, R. y SALVADOR, F. (2006). El proceso de planificación en la expresión escrita de alumnos sordos: estudio de casos en Educación Secundaria. Revista de Educación, 339, 435-453.

HAYES, J. R. (1996). A new framework for understanding cognition and affect in writing. En C. M. Levy y S. R. Ransdell (eds.), The Science of Writing. Hillsdale, NJ: Lawrence Erlbaum Associates, pp. 1-27.

HAYES, J. R. y NASH, J. (1996). On the nature of planning in writing. En C. M. Levy y S. R. Ransdell (eds.), The Science of Writing. Hillsdale, NJ: Lawrence Erlbaum Associates, pp. 29-55.

HEDGE, T. (1998). Writing. A Resource Book for Teachers. Oxford: Oxford University Press.

HODDER, I. (2000). The interpretation of documents and material culture. En N. K. Denzin y Y. S. Lincoln. (eds.): Handbook of Qualitative Research. London: Sage Publications, pp. 703-717. 
JONES, S. y MYHILL, D. (2007). Discourses of difference? Examining gender differences in linguistic characteristics of writing. Canadian Journal of Education, 30(2), 456-482.

KRIPPENDORFF, K. (2002). Metodología de análisis de contenido. Teoría y práctica. Barcelona: Paidós.

LECUONA, M. P. (Dir.) (1999). Pensar para escribir. Un programa de enseñanza para la composición escrita. Madrid: CIDE.

LECUONA, M. P.; RODRÍGUEZ, M. J. y SÁNCHEZ, M. C. (2003). Evaluación de modelos de composición escrita en Educación Primaria. Revista de Educación (Madrid), 332, 301-326.

MCCARTHUR, C. A.; GRAHAM, S. y FITZGERALD, J. (2006). Handbook of writing research. New York: Guilford Press.

MCMILLAN, J. H. y SCHUMACHER, S. (2005). Investigación educativa. Una introducción conceptual (5 $5^{\mathrm{a}}$ ed.). Madrid: Pearson.

MILLARD, E. (1997). Differently literate: Boys, girls and the schooling of literacy. London: Falmer.

MIRANDA, A.; GARCÍA, R. y SORIANO, M. (2005). Habilidad narrativa de los niños con trastorno por déficit de atención con hiperactividad. Psicothema, 17(2), 227-232.

PETERSON, S. y KENNEDY, K. (2006). Sixth grade teachers' written comments on student writing: genre and gender influences. Written Communication, 23(1), 36-62.

RAMOS, J. L.; CUADRADO, I. y IGLESIAS, B. (2005). La composición escrita en el alumnado de Educación Primaria y Secundaria. Cultura y educación, 17(3), 239-251.

RICHARDSON, V. (2001). Handbook of research on teaching. Washington: American Educational Research Association.

SALVADOR, F. (2000). Habilidades narrativas de alumnos de Educación Primaria en la producción de textos escritos. Enseñanza, 17-18, 145-163.

SALVADOR, F. (2005). La expresión escrita de alumnos con necesidades educativas especiales. Procesos cognitivos. Málaga: Aljibe.

SALVADOR, F. (2008). Psicopedagogía de la lengua escrita. Madrid: EOS.

SALVADOR, F. y GARCÍA, I. (2007). Procesos cognitivos en la composición escrita de personas afectadas de parálisis cerebral. Innovación Educativa, 17, 229-238.

SMITH, F. (1982). Writing and the writer. Portsmouth, NH: Heinemann.

STAKE, R. (1998). Investigación con estudio de casos. Madrid: Morata.

TRACY, B.; REID, R. y GRAHAM, S. (2009). Teaching Young Students Strategies for Planning and Drafting and Drafting Stories: The Impact of Self-Regulated Strategy Development. The Journal of Educational Research, 102(5), 323-332.

TRIBBLE, CH. (1996). Writing. Oxford: Oxford University Press.

TROIA, G. A. (2002). Writing models: strategies for writing composition in inclusive settings. Reading and Writing Quarterly: Overcoming Learning Difficulties, 17(3), 243-248. 
VILÀ, M. (1998). Un estudio de casos sobre el proceso de composición escrita en chicos y chicas de ciclo superior de E.G.B. en Gerona. En R. Pérez (coord.), Educación y Diversidad II (pp. 743-766). Oviedo: Universidad de Oviedo.

WONG, B. Y. L.; BUTLER, D. L.; FICZERE, S. A. y KUPERIS, S. (1997). Teaching Adolescents with Learning Disabilities and Low Achievers to Plan, write Revise Contrast Essays. Learning Disabilities Research and Practice, 12(1), 2-15. 